Göran Hammarström

\title{
Comment on Dumitru Cornel Vilcu's contribution
}

Vilcu, philosophers and others have difficulty understanding time. It is, however, fortunate for our daily life that most people do not have any such difficulty. Through their language they have perfect knowledge of time and they handle it without any problem: It takes time to do things. There are points in time which are situated in the past, present and future. Things can begin and end and have a longer or shorter time in between. So they last a longer or a shorter time. Things that happen can be slow or fast. One can measure time in seconds, minutes, hours, days, weeks, months and years. There is nothing unclear or problematic about these things. People understand time without any difficulty but great philosophers and anybody trying to define time have serious problems. Vilcu discusses the notion of time quoting philosophers but I cannot see that they deal correctly with the basic problem. Time is a notion that refers to a certain aspect of processes (developments, changes). This aspect can even be exactly measured. Processes can be observed. The life of a dog is a process from birth to death. A human has a similar process but it takes a much longer time. As far as I can understand, the discussions of time are usually inadequate because one does not consider the basic thing, the development aspect of processes, which is what people estimate or even measure using a clock. The time of is based on another process, the sun going up and down. These estimates or the more exact measures are what the notion of time refers to. Vilcu discusses time on the basis of Bergson's ideas (p. 4): “time only appears where some kind/degree of biological liberty manifests itself”. He says that humans can apply time to the movement of a pendulum. But this time is then not considered to be what time really is because "the pendulum itself does not grow older, it knows nothing of change. It does not move through time". I maintain that this thinking is incorrect because time is wrongly understood. It should only refer to a process and the definition cannot include certain things such as aspects of what is estimated or measured. The same estimation or exact measure of time must apply to the movement of the pendulum and to the changes of "biological" beings. Vilcu says further (p. 4): "Time does not exist for a grain of sand moved by the winds but 'it is there' for a thistle clinging to a rock". This is strange. None of the two has a notion of time but a thinking human can use time for both. He can say that the grain of sand has been moved by the wind for an hour and the life time of the thistle is a year. The difference is that the thistle has life which is a process to which the notion of time can be applied but we believe that the grain of sand does not change. Since time is a human notion, it goes without saying that animals and lifeless things cannot possess this notion although it can be applied to them.

Vilcu says (p. 3) after having provided details of human life, that time "dominates us, never stops passing, and carry us with its flow". He is referring to facts such as being "young and playful" and "old and bitter". I can, however, not remember having heard somebody saying anything that implies that he is dominated by time. What humans are basically dominated by are such things as the state of their health, feeling young and strong, feeling old and weak or regretting no longer being young. That such things are sometimes 
clearly thought of as being related to the notion of time is true but this is not usually people's basic concern. Their most important concerns are related to states and processes such as all the details of health, feelings and states of mind.

May I try to explain my own understanding of time? Time is one of three fundamental dimensions we deal with in our daily life, the two others being length and weight. When one tries to understand the two latter I do not think anybody includes examples of what they can be applied to and what we feel about them although adding examples may clarify the definition. In regard to understanding time Vilcu and some philosophers include examples in the basic definition. Time is then not the same if one estimates or measures the process of a moving pendulum or that of the life of a human. I believe that this is wrong. The time, whether it is estimated or measured, is the same in both cases. What is exactly time? Time is a developmental aspect of processes which we can estimate or measure. These have a beginning point and an end point. Time is the length between the two points mentioned. But points of beginning and end can refer to many things. Yes, but here they are points in time (ahem). And length can refer to the length of a stick. Yes, but here it refers to the length of time (ahem). So the definition is circular. Yes, but if this is the best one can do, the circle is not vicious. It must be accepted and it is perfect for our understanding of time. (If one can observe the two points in time, not only the time of a process but also the time of a state can be estimated or measured.) A short definition of time (see Google) is that it is duration. Yes, and duration is the time between two points in time (ahem).

Some people and some philosophers cannot solve the problem of time. The problem is that there is no problem. Humans understand things in complicated ways through their language. When one wants to understand the meaning of words the explanations often have to be circular. This circumstance does not prevent linguists from writing monolingual dictionaries which have many circular definitions. People in general and scientists use the fundamental internationally accepted measuring system centimetre - gram - second without bothering about a not existing problem. Nobody finds that a distance is different if one considers the unconscious road and the conscious human travelling on it. And nobody finds that the weight is different if one considers a piece of unconscious rock and a human lifting it. So why is time different when used about a pendulum or a grain of sand and about a human being?

While time and the related notion of order are used at the description of various levels of a language, the notion of time itself does not create any problems. It does not really need to be discussed by a linguist. My critique of various ways of understanding time discussed above is that they are formulated as being definitions of time: "time is..." while being all kinds of perceptions and ideas which possess an element of time (being alive, growing old, feeling young). These can be partly defined by using time but they have wrongly been used for the definition of time, which is something like: a stretch of a process between a beginning point and an end point. (Hidden circularity? Yes, I know.) Time can be estimated or it can be measured by a clock. 